\title{
STUDY OF LIVER FUNCTION PARAMETERS IN DENGUE INFECTION
}

\author{
Hareesh Rangaswamaiah', Srinivasa Kaligonahalli Venkataramanappa², Rekha Gangadharappa 3 , Mikita Arun Suvarna
}

${ }^{1}$ Associate Professor, Department of General Medicine, Dr. B. R. Ambedkar Medical College and Hospital, Bengaluru. ${ }^{2}$ Associate Professor, Department of General Medicine, Dr. B. R. Ambedkar Medical College and Hospital, Bengaluru. ${ }_{3}^{3}$ Postgraduate Student, Department of General Medicine, Dr. B. R. Ambedkar Medical College and Hospital, Bengaluru. ${ }_{4}^{4}$ Postgraduate Student, Department of General Medicine, Dr. B. R. Ambedkar Medical College and Hospital, Bengaluru.

ABSTRACT

\section{BACKGROUND}

Dengue fever is the most common arthropod-borne arboviral disease in humans. Globally, 2.5-3 billion individuals live in approximately 112 countries that experience dengue transmission. Annually, approximately 50-100 million individuals are infected. This study was done to assess the correlation between liver function parameters and the severity of the disease as well as to gauge whether they are good predictors of morbidity and mortality in case of dengue fever.

\section{MATERIALS AND METHODS}

A retrospective and descriptive study was undertaken among inpatients in a tertiary care hospital. The data of 561 patients was collected and analysed. All patients who were seropositive for dengue were included in the study. Clinical features, haematological and biochemical parameters as well as ultrasonographic findings were noted.

\section{RESULTS}

Of the 561 patients studied, majority were in the age group of 26 to 30 years (266 or $47.42 \%$ ) and were males (411 or $73.26 \%$ ). Fever was the most common presentation (100\%). Headache and myalgia were seen in majority of cases. Retro-orbital pain was noticed in only in $4.99 \%$ of cases. 278 (49.55\%) patients had fever with gastrointestinal symptoms. 37 (6.59\%) patients had bleeding manifestations, $8(1.43 \%)$ patients had clinical jaundice. $348(62.03 \%)$ patients had Aspartate aminotransferase levels within normal range, 107 (19.07\%) patients had levels 3 times the upper limit, 106 (18.89\%) had levels 3-10 times the upper limit. 351 (62.56\%) patients had Alanine aminotransferase levels within normal range, 84 (14.97\%) patients had levels 3 times the upper limit, 126 (22.45\%) had levels 3-10 times the upper limit. None of the study population had levels $>10$ times the normal. Mortality was $0.53 \%$.

\section{CONCLUSION}

Dengue viral fever can be suspected when there is a fever, associated with headache, myalgia, retro-orbital pain, erythematous morbilliform rash, conjunctival suffusion and itching in palms and soles along with thrombocytopenia, leucopenia and raised liver transaminases enzymes. Liver function tests are very good parameters to assess the severity, mortality and outcome of dengue fever. Early diagnosis, careful monitoring of clinical, biochemical and blood parameters as well as proper fluid management goes a long way in reducing the mortality of dengue fever.

\section{KEYWORDS}

Alanine Aminotransferase, Aspartate Aminotransferase, NS1-Non-Structural Protein-1.

HOW TO CITE THIS ARTICLE: Rangaswamaiah H, Venkataramanappa SK, Gangadharappa R, et al. Study of liver function parameters in dengue infection. J. Evolution Med. Dent. Sci. 2017;6(15):1220-1225, DOI: 10.14260/Jemds/2017/265

\section{BACKGROUND \\ Dengue is an acute febrile disease. The aetiologic agent of dengue is the dengue virus (DENV), which belongs to the Flaviviridae family and the Flavivirus genus and has four serotypes, transmitted by Aedes aegypti as well as Aedes albopictus. Dengue is the most common arthropod-borne viral disease and is one of the most significant diseases because it is associated with high rates of morbidity and mortality. ${ }^{1-3}$}

Financial or Other, Competing Interest: None.

Submission 15-12-2016, Peer Review 04-02-2017,

Acceptance 10-02-2017, Published 20-02-2017.

Corresponding Author:

Dr. Hareesh Rangaswamaiah

Associate Professor,

Department of General Medicine,

DR. B. R. Ambedkar Medical College,

K. G. Halli,

Bengaluru-560045.

E-mail: hareesh22578@gmail.com

DOI: $10.14260 /$ jemds $/ 2017 / 265$
Although the majority of dengue infections result in asymptomatic infection or manifest as undifferentiated viral fever, some develop fluid leakage and bleeding manifestations which result in dengue haemorrhagic fever (DHF) and dengue shock syndrome (DSS). ${ }^{4}$

\section{Case Definition}

Dengue has been traditionally classified into dengue fever (DF), DHF and DSS (WHO 1997 Classification).

The modified categorisation of WHO in 2009 includes dengue with or without warning signs or severe dengue.

Criteria for Dengue \pm Warning Signs (Who 2009)

Probable Dengue

Live in /travel to dengue endemic area.

\section{Fever and 2 of the following Criteria}

- Nausea, vomiting.

- Rash.

- Aches and pains.

- Tourniquet test positive. 
- Leukopenia.

- Any warning sign.

\section{Warning Signs}

- Abdominal pain or tenderness.

- Persistent vomiting.

- Clinical fluid accumulation.

- Mucosal bleed.

- Lethargy, restlessness.

- Liver enlargement $>2 \mathrm{~cm}$.

- Laboratory: Increase in HCT concurrent with rapid decrease in platelet count.

\section{Criteria for Severe Dengue}

Severe Plasma Leakage Leading to

- Shock (DSS).

- Fluid accumulation with respiratory distress.

\section{Severe Bleeding}

- As evaluated by clinician.

Severe Organ Involvement

- Liver: AST or ALT >=1000.

- CNS: Impaired consciousness.

- Heart and other organs.

Criteria for Dengue \pm Warning Signs

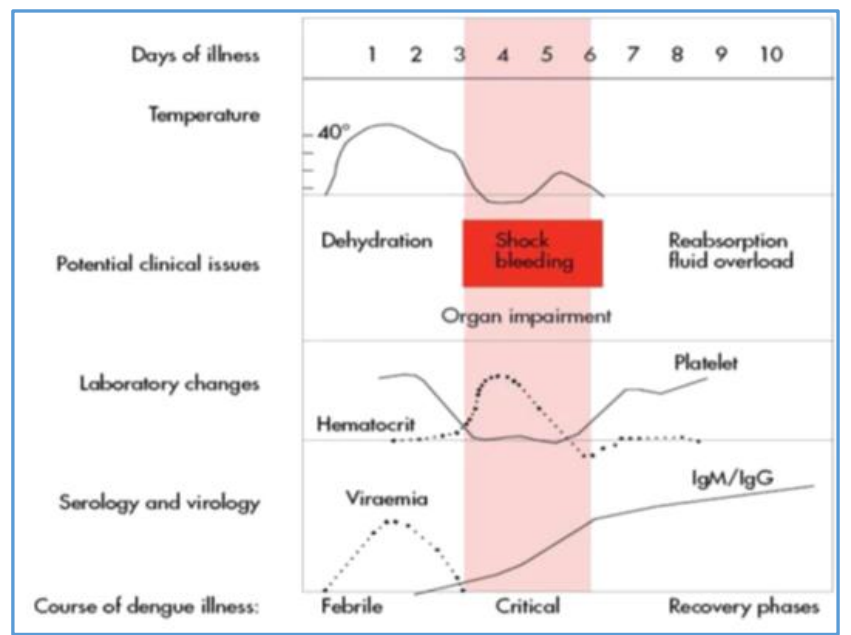

Figure 1. Dengue Disease Phases and Potential Complications. Reproduced, with Permission of the Publisher, from the WHO Publication[5] (Figure 2.1; p. 25)

Detection of the dengue virus by virus isolation or by nucleic acid detection methods are considered as confirmatory tests for confirming the diagnosis of dengue infection. 6

MAC-ELISA (IgM- Capture Enzyme-Linked Immunosorbent Assay) has become widely used test in the past few years. It is simple and based on detection of dengue specific IgM antibodies. An indirect IgG-ELISA has been developed to detect primary and secondary dengue infections. ${ }^{7}$

So many commercial tests are available for the detection of dengue NS1 antigen, which is a non-structural protein of the dengue virus. ${ }^{8}$

\section{Dengue and Liver}

A high level of viral load is associated with involvement of different organs (liver, brain) in the severe form of the dengue viral disease. ${ }^{9}$ Hepatic dysfunction is demonstrated by hepatomegaly and mild-moderate increases in the transaminase levels although jaundice and acute liver failure are generally uncommon. ${ }^{10-13}$

Dengue fever initiates inflammatory responses leading to a liver parenchymal lesion that releases these markers into the blood. 13

In approximately $90 \%$ of the patients with dengue fever, Aspartate aminotransferase (AST) is higher than the Alanine aminotransferase (ALT). ${ }^{14}$

\section{Degree of Liver Damage}

The degree of liver damage was classified into four groups according to AST and ALT levels during the period of infection.

Grade A: Patients with normal AST and ALT levels. The laboratory reference values of AST and ALT for males were $59 \mathrm{UI} / \mathrm{L}$ and $72 \mathrm{UI} / \mathrm{L}$, respectively, and for women reference values were $36 \mathrm{IU} / \mathrm{L}$ and $52 \mathrm{IU} / \mathrm{L}$, respectively.

Grade B: Patients in whom the level of at least one of the aminotransferases was increased but no higher than three times the normal value.

Grade C: One of the enzymes were between 3 and 10 times the reference values.

Grade D: An increase in one or both enzymes to levels more than 10 times the reference values. ${ }^{13}$

\section{MATERIALS AND METHODS}

This study is a retrospective descriptional study done in Dr B. R. Ambedkar Medical College and Hospital.

\section{Study Sample}

A total of 561 suspected dengue seropositive cases included in this study between November 2015 and October 2016 at Dr. B R Ambedkar Medical College and Hospital.

Dengue was suspected when two or more of the following symptoms were present: fever, headache, retroorbital pain, myalgia, joint pain, skin rash, nausea, vomiting, prostration and haemorrhagic manifestations.

Patients were either treated as outpatients or as inpatients in our institute in accordance with the standard protocol during the period in which symptoms were present or while laboratory tests remained abnormal. Complete blood counts and liver function tests were carried out and monitored.

\section{Serology}

The blood samples were simultaneously submitted to serological tests: immunoenzymatic assay of NS1, IgMDengue, IgG-Dengue. When the results of NS1 or NS1 and IgM tests were positive, patients were considered to be currently infected by the dengue virus, while cases in which the results of samples were positive for only IgG are considered remote infection. 


\section{Inclusion Criteria}

All adults $>18$ years who present to OP/IP with clinical symptoms of dengue fever/DHF/DSS who are seropositive are included in the study.

\section{Exclusion Criteria}

1. Patients who have history of chronic ethanol consumption.

2. Active Hepatitis A, B, C, E infection.

3. Patients who are on hepatotoxic drugs.

\section{RESULTS}

This study clinical data was taken retrospectively from patients receiving care as in-patients in Dr. B R Ambedkar Medical College and Hospital from November 2015 to October 2016.

A total number of 561 patients were studied.

\section{Age Distribution}

\begin{tabular}{|c|c|}
\hline Age Distribution & Number of Patients \\
\hline Age Distribution & 128 \\
\hline $18-25$ years & 266 \\
\hline $26-30$ years & 40 \\
\hline $31-35$ years & 36 \\
\hline $36-40$ years & 91 \\
\hline$>40$ years & $\mathbf{5 6 1}$ \\
\hline Total
\end{tabular}

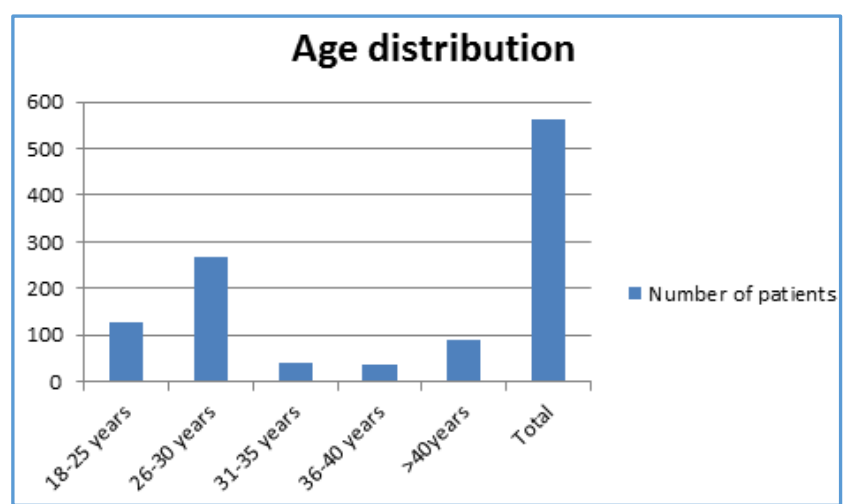

Figure 1. Age Distribution

Commonly involved age group was between 26 to 30 years.

\section{Sex Distribution}

Among the study population, 411 (73.13\%) were male patients and $150(26.69 \%)$ were female patients.

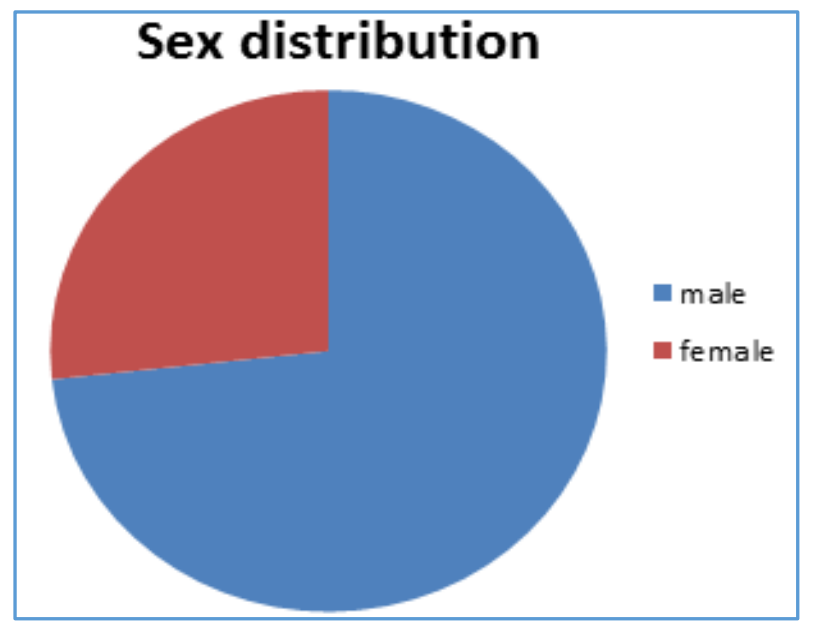

Figure 2. Sex Distribution
Clinical Profile

\begin{tabular}{|c|c|}
\hline Symptoms & Number of Patients \\
\hline Fever & 10 \\
\hline Fever, headache, polyarthralgia & 113 \\
\hline Fever, rash & 5 \\
\hline Fever, retro-orbital pain & 28 \\
\hline Fever with gastrointestinal symptoms & 278 \\
\hline Fever with bleeding manifestations & 37 \\
\hline Clinical jaundice & 8 \\
\hline
\end{tabular}

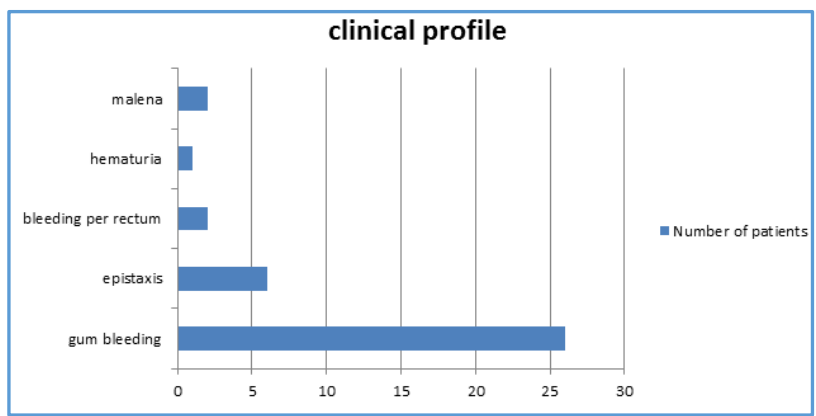

Figure 3. Clinical Profile

The major symptoms among the study population are fever with gastrointestinal symptoms (nausea, vomiting, pain abdomen, loose stools). 37 patients had bleeding manifestations, 8 patients had clinical jaundice.

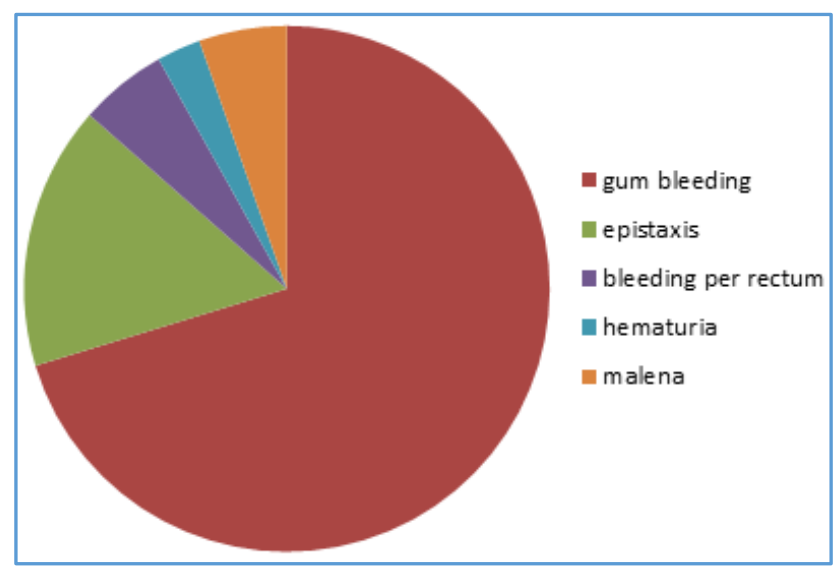

Figure 4. Bleeding Manifestations

\begin{tabular}{|c|c|c|}
\hline Sl. No. & Clinical Dengue & Number of Patients \\
\hline 1. & Probable dengue & $235(41.89 \%)$ \\
\hline 2. & Dengue with warning signs & $323(57.57 \%)$ \\
\hline 3. & Severe dengue & $3(0.5 \%)$ \\
\hline
\end{tabular}

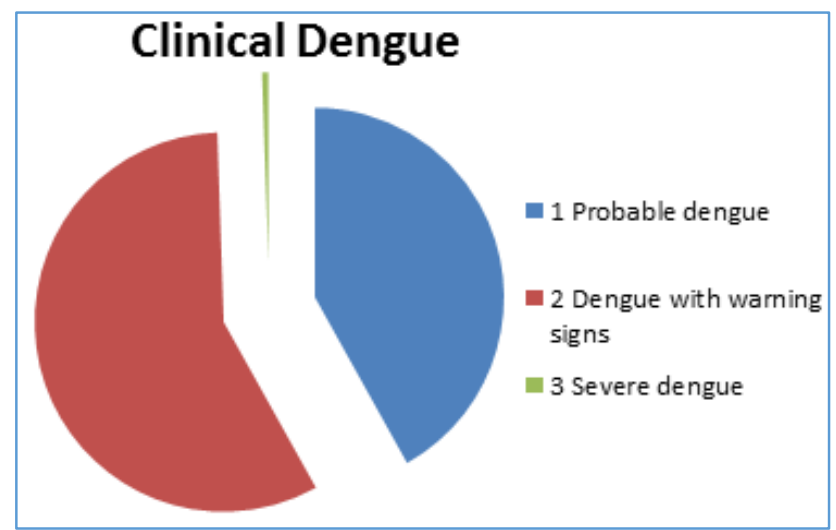


According to WHO clinical criteria for dengue, out of 561 patients in our study, 235 (41.89\%) were probable dengue, 323 (57.57\%) were Dengue with warning signs and $3(0.5 \%)$ had Severe dengue. Patients in severe dengue criteria had $100 \%$ mortality in our study.

\section{Serology}

\begin{tabular}{|c|c|}
\hline Serology Positivity & Number of Patients \\
\hline Dengue NS1, IgM, IgG positive & 9 \\
\hline Dengue NS1 positive & 346 \\
\hline Dengue IgM, IgG positive & 153 \\
\hline Dengue IgM positive & 43 \\
\hline Dengue IgG positive & 10 \\
\hline
\end{tabular}

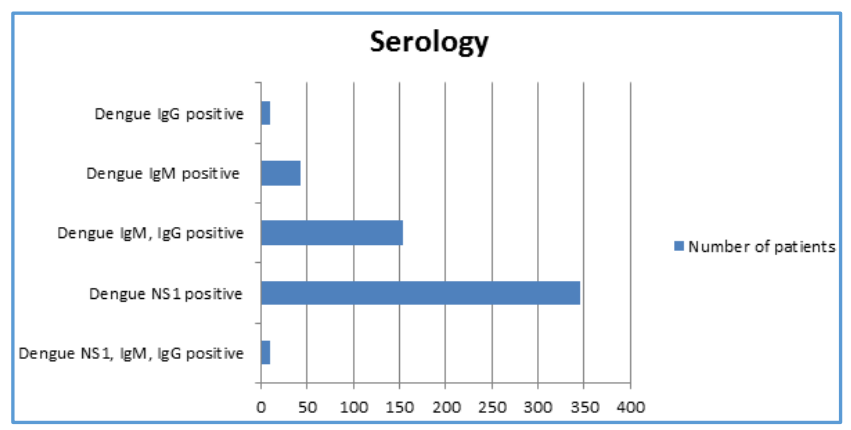

Figure 6. Serology

346 patients were positive for Dengue NS1 antigen; 153 were Dengue NS1, IgM, IgG positive; 43 patients were dengue IgM positive.

\section{Liver Function Parameters}

\section{Aspartate Aminotransferase Levels}

\begin{tabular}{|c|c|c|c|}
\hline AST & Male & Female & Total \\
\hline Grade A & 259 & 89 & $348(62.03 \%)$ \\
\hline Grade B & 93 & 14 & $107(19.07 \%)$ \\
\hline Grade C & 59 & 47 & $106(18.89 \%)$ \\
\hline Grade D & 0 & 0 & $0(0 \%)$ \\
\hline Total & $\mathbf{4 1 1}$ & $\mathbf{1 5 0}$ & $\mathbf{5 6 1 ( 1 0 0 \% )}$ \\
\hline
\end{tabular}

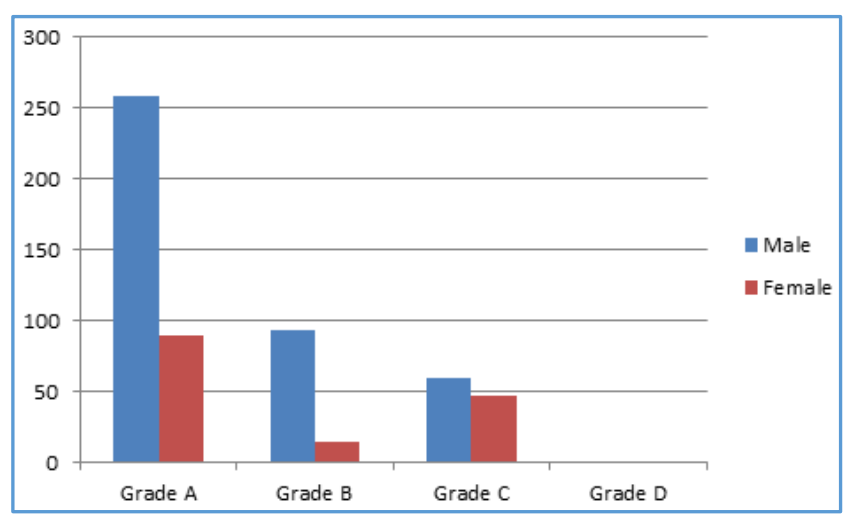

Figure 7. AST levels in Study Population

348 (62.03\%) patients had Aspartate aminotransferase levels within normal range, 107(19.07\%) patients had levels 3 times the upper limit, 106(18.89\%) had levels 3-10 times the upper limit. None of the study population had levels $>10$ times the normal.
Alanine Aminotransferase Levels

\begin{tabular}{|c|c|c|c|}
\hline ALT & Male & Female & Total \\
\hline Grade A & 258 & 93 & $351(62.56 \%)$ \\
\hline Grade B & 65 & 19 & $84(14.97 \%)$ \\
\hline Grade C & 88 & 38 & $126(22.46 \%)$ \\
\hline Grade D & 0 & 0 & $0(0 \%)$ \\
\hline Total & $\mathbf{4 1 1}$ & $\mathbf{1 5 0}$ & $\mathbf{5 6 1 ( 1 0 0 \% )}$ \\
\hline
\end{tabular}

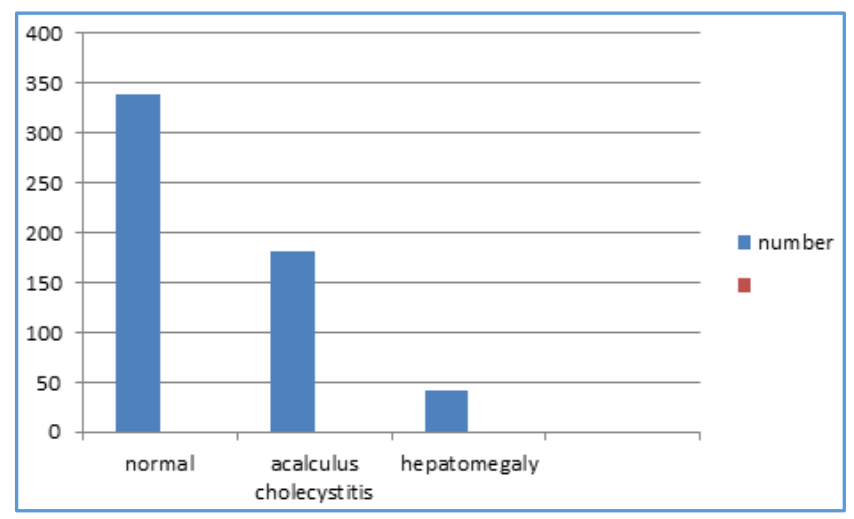

Figure 8. ALT levels in Study Population

351 (62.56\%) patients had Alanine aminotransferase levels within normal range, $84(14.97 \%)$ patients had levels 3 times the upper limit, 126 (22.45\%) had levels 3-10 times the upper limit. None of the study population had levels $>10$ times the normal.

\section{Ultrasonography Abdomen Findings}

338 patients had no sonological abnormalities, 182 patients had acalculous cholecystitis and 41 patients had hepatomegaly.

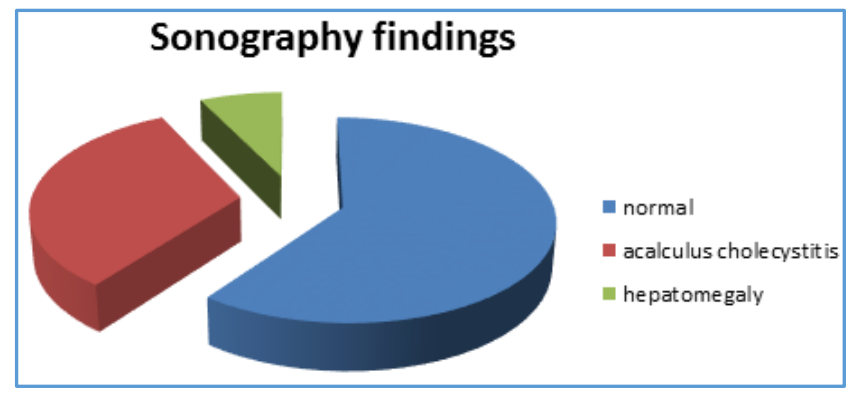

Figure 9. Ultrasonography Abdomen Findings

\section{Duration of Hospital Stay}

\begin{tabular}{|c|c|c|c|}
\hline $\begin{array}{c}\text { Grade of } \\
\text { Transaminitis }\end{array}$ & $\begin{array}{c}<\mathbf{7 2} \\
\text { Hours }\end{array}$ & $\begin{array}{c}\mathbf{> 2} \\
\text { Hours }\end{array}$ & Total \\
\hline A & 312 & 39 & 351 \\
\hline B & 52 & 32 & 84 \\
\hline C & 22 & 104 & 126 \\
\hline D & 0 & 0 & 0 \\
\hline Total & $\mathbf{3 8 6}$ & $\mathbf{1 7 5}$ & $\mathbf{5 6 1}$ \\
\hline
\end{tabular}




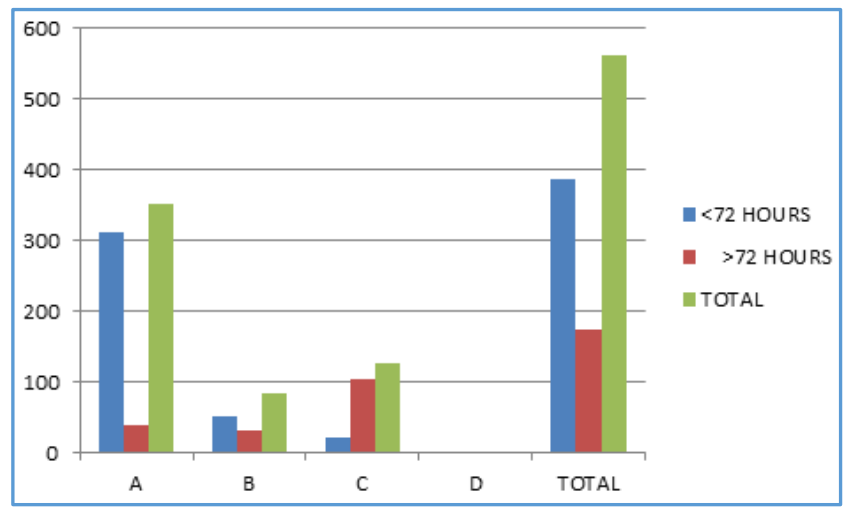

Figure 10. Duration of Hospital Stay

Duration of hospital stay was longer for patients belonging to group C. 3 deaths secondary to dengue shock syndrome were in group $\mathrm{C}$.

\section{DISCUSSION}

Increase in the number of dengue cases over the past few years has been attributed to rapid urbanisation with unchecked constructions and poor sanitation facilities contributing fertile breeding areas for mosquitoes, it is also seen that increase in alertness among medical personnel following the epidemics and availability of diagnostic tools in the hospitals have contributed to the increased detection of cases. ${ }^{15}$

In this study, common age group was between 26 to 30 years. Male to female ratio in our study was 2.74:1. Rajesh Deshwal et al study also showed male to female ratio was 2.67:1.

Fever was the most common presentation (100\%), which is similar to other studies from India and South East Asia. 16-19 Headache and myalgia were seen in majority of cases. Retroorbital pain was noticed in only in $4.99 \%$ of cases.

The next major symptoms are gastrointestinal symptoms (nausea, vomiting, pain abdomen, loose stools). 278 (49.55\%) patients had fever with gastrointestinal symptoms. 37 (6.59\%) patients had bleeding manifestations, 8 (1.43\%) patients had clinical jaundice.

Jayanta Samanta et al study also had demonstrated clinical jaundice in $1.7-17 \%$ of patients. Hepatomegaly is present in both probable dengue and dengue fever with warning signs, more common with the latter; 41 patients (7.3\%).

$182(32.44 \%)$ patients had acalculous cholecystitis in our study. Acalculous cholecystitis is a very known entity among the patients with dengue fever but none of them have shown comparison according to severity of hepatitis. 20 ,

The extent to which the liver is affected by the dengue virus ranges from mild lesions to fulminant hepatitis. Liver involvement may be characterised by manifestations such as pain in right hypochondrium, hepatomegaly, varying degree of jaundice and increase in liver markers principally ALT and AST similar to those found in acute hepatitis caused by Hepatitis A, B, C, D and E viruses. ${ }^{21,22,23}$

In our study, 348 (62.03\%) patients had aspartate aminotransferase levels within normal range, 107 (19.07\%) patients had levels 3 times the upper limit, 106 (18.89\%) had levels 3-10 times the upper limit. 351 (62.56\%) patients had alanine aminotransferase levels within normal range, 84
(14.97\%) patients had levels 3 times the upper limit, 126 (22.45\%) had levels 3-10 times the upper limit. None of the study population had levels $>10$ times the normal.

\section{CONCLUSION}

Dengue fever can be suspected when there is fever associated with headache, retro-orbital pain, erythematous morbilliform rash, conjunctival suffusion and itching in palms and soles along with thrombocytopenia, leucopenia and elevated liver transaminases. Liver function tests are very good parameters to assess the severity, mortality and outcome of dengue fever. Early diagnosis, careful monitoring of clinical, biochemical and blood parameters as well as proper fluid management goes a long way in reducing the mortality of dengue fever.

\section{REFERENCES}

[1] De Souza LJ, Alves JG, Nogueira RMR, et al. Aminotransferase changes and acute hepatitis in patients with Dengue fever: analysis of 1,585 cases. Braz J Infect Dis 2004;8(2):153-63.

[2] de Souza LJ, Carneiro HG, Filho SJT, et al. Hepatitis in Dengue shock syndrome. Braz J Infect Dis 2002;6(6):322-7.

[3] Hammond NS, Balmaseda A, Perez L, et al. Differences in Dengue severity in infants, children and adults in a 3year hospital-based study in Nicaragua. Am J Trop Med Hyg 2005;73(6):1063-70.

[4] Malavige GN, Ogg GS. T cell responses in Dengue viral infections. J Clin Virol 2013;58(4):605-11.

[5] Dengue guidelines for diagnosis, treatment, prevention and control. http://www.who.int/rpc/guidelines/9789241547871/ en/.

[6] WHO. Comprehensive guidelines for prevention and control of Dengue fever and dengue haemorrhagic fever. In Geneva, Switzerland: World Health Organization, 2011.

[7] Guidelines for clinical management of Dengue fever. Dengue hemorrhagic fever and Dengue shock syndrome- Directorate of NVBDCP. 2008:11-12.

[8] De Costa VG, Marques-Silva AC, Moreli ML. A metaanalysis of the diagnostic accuracy of two commercial NS1 antigen ELISA tests for early Dengue virus detection. PLoS One 2014;9(4):e94655.

[9] Marianneau P, Steffan AM, Royer C, et al. Infection of primary cultures of human Kupffer cells by Dengue virus: no viral progeny synthesis, but cytokine production is evident. J Virol 1999;73(6):5201-6.

[10] Kuo CH, Tai DI, Chang-Chien CS, et al. Liver biochemical tests and Dengue fever. Am J Trop Med Hyg 1992;47(3):265-70.

[11] Nguyen TL, Nguyen TH, Tieu NT. The impact of Dengue haemorrhagic fever on liver function. Res Virol 1997;148(4):273-7.

[12] Mohan B, Patwari AK, Anand VK. Hepatic dysfunction in childhood dengue infection. J Trop Pediatr 2000;46(1):40-3.

[13] De Souza LJ, Nogueira RM, Soares LC, et al. The impact of dengue on liver function as evaluated by aminotransferase levels. Braz J Infect Dis 2007;11(4):407-10. 


\section{Jemds.com}

[14] Kalayanarooj S, Vaughn DW, Nimmanithya S, et al. Early clinical and laboratory indicators of acute Dengue illness. J Infect Dis 1997;176(2):313-21.

[15] Gubler DJ. Dengue and Dengue hemorrhagic fever. Clin Microbiol Rev 1998;11(3):480-96.

[16] Srikiatkhachorn A, Gibbons RV, Green S, et al. Dengue hemorrhagic fever: the sensitivity and specificity of the world health organization definition for identification of severe cases of Dengue in Thailand, 1994-2005. Clin Infect Dis 2010;50(8):1135-43.

[17] Kashinkunti MD, Shiddappa, Dhananjaya M. A study of clinical profile of dengue fever in a tertiary care teaching hospital. Sch J App Med Sci (SJAMS) 2013;1(4):280-2.

[18] Daniel R, Rajamohanan, Philip AZ. A study of clinical profile of Dengue fever in Kollam, Kerala, India. Dengue Bulletin 2005;29:197-202.

\section{Original Research Article}

[19] Munde DD, Shetkar UB. Clinical features and haematological profile of Dengue fever. Indian J Appl Res 2013;3(1):131-2.

[20] Sharma N, Mahi S, Bhalla A, et al. Dengue fever related acalculous cholecystitis in a north Indian tertiary care hospital. J Gastroenterol Hepatol. 2006;21(4);664-7.

[21] Wu KL, ChangChien CS, Kuo CM, et al. Dengue fever with acute acalculous cholecystitis. Am J Trop Med Hyg 2003;68(6):657-60.

[22] Lum LC, Lam SK, George R, et al. Fulminant Hepatitis in Dengue infection. South-East Asian J Trop Med Public Health 1993;24(3):467-71.

[23] Wang LY, Chang WY, Lu SN, et al. Sequential changes of serum transaminases and abdominal sonography in patients with suspected dengue fever. Gaoxiong Yi Xue Ke Xue Za Zhi 1990;6(9):483-9. 